\title{
NOninvasive Monitoring for Early Detection of Atrial Fibrillation: rationale and design of the NOMED-AF study
}

\author{
Zbigniew Kalarus ${ }^{1,2}$, Paweł Balsam³, Piotr Bandosz ${ }^{4}$, Tomasz Grodzicki ${ }^{5}$, Jarosław Kaźmierczak ${ }^{6}$, \\ Radosław Kiedrowicz ${ }^{6}$, Katarzyna Mitręga ${ }^{1}$, Michał Noczyński ${ }^{7}$, Grzegorz Opolski ${ }^{3}$, Krzysztof Rewiuk ${ }^{5}$, \\ Marcin Rutkowski ${ }^{4}$, Adam Sokal ${ }^{1}$, Beata Średniawa ${ }^{1,2}$, Łukasz Wierucki ${ }^{4}$, Michał Wiśniewski ${ }^{8}$, \\ Tomasz Zdrojewski ${ }^{4}$, Gregory Y.H. Lip ${ }^{9}$ \\ 'Department of Cardiology, School of Medicine with the Division of Dentistry in Zabrze, Medical University of Silesia, Katowice, Poland \\ 2Silesian Park of Medical Technology Cardio-Med Silesia, Zabrze, Poland \\ ${ }^{3} 1^{\text {st }}$ Chair and Department of Cardiology, Medical University of Warsaw, Warsaw, Poland \\ ${ }^{4}$ Department of Preventive Medicine and Education, Medical University of Gdansk, Gdansk, Poland \\ ${ }^{5}$ Department of Internal Medicine and Gerontology, Jagiellonian University Medical College, Krakow, Poland \\ ${ }^{6}$ Department of Cardiology, Pomeranian Medical University, Szczecin, Poland \\ ${ }^{7}$ Comarch Healthcare SA, Krakow, Poland \\ ${ }^{8}$ Comarch SA, Krakow, Poland \\ ${ }^{9}$ Institute of Cardiovascular Sciences, University of Birmingham, Birmingham, United Kingdom
}

\section{INTRODUCTION}

Atrial fibrillation (AF) is the most common arrhythmia worldwide, affecting $1 \%$ to $2 \%$ of the general population, with an increasing prevalence and incidence in the elderly [1-3]. "Silent AF" is diagnosed in individuals who are asymptomatic and is usually discovered based on incidental electrocardiographic (ECG) testing $[4,5]$. The frequency of silent AF in the general population is less certain, ranging from $1 \%$ to $10 \%$, and is deemed the cause of $4.4 \%$ of all episodes of ischaemic stroke $[1,6]$. While long-term ECG monitoring allows early detection of AF in larger groups of individuals compared with short-term ECG recordings, there are currently no registered or approved non-invasive systems for heart rhythm monitoring for long-term use.

The aims of the NOMED-AF study are to determine the prevalence of $\mathrm{AF}$ in the Polish population using long-term ECG monitoring in individuals aged $\geq 65$ years, and to ascertain the independent risk factors for the clinically symptomatic and asymptomatic forms of AF.

\section{METHODS}

The NOMED-AF study is a cross-sectional, observational study aimed at the assessment of the prevalence of AF and its correlation with cardiovascular disease risk factors in the Polish population, based on a representative sample of adults aged $\geq 65$ years. The main objective of the survey is to evaluate the prevalence of $\mathrm{AF}$ and the associated comorbidities.

The assessment of AF prevalence will be performed with the use of an ECG monitoring system developed and validated during the first and second phases of the project as well as a detailed questionnaire, follow-up data sheet, blood pressure (BP) measurements, and blood/urine sample collection.

An outline of the study protocol is shown in Figure 1 and Table 1A. The study was approved by the Local Bioethical Committee (26/2015) and is registered on clinicaltrials.gov (NCT03243474; https://clinicaltrials. gov/ct2/show/NCT03243474). Enrolment began on March 15, 2017, and the study was registered on August 9, 2017. For further details see Supplement on-line (see journal website).

\section{Study population}

A total of 3000 individuals (both men and women) are planned to be enrolled in this part of the study. Unselected individuals aged $\geq 65$ years will be included; the only exclusion criterion is age $<65$ years.

\section{Address for correspondence:}

Prof. Zbigniew Kalarus, Department of Cardiology, School of Medicine with the Division of Dentistry in Zabrze, Medical University of Silesia,

ul. Curie-Skłodowskiej 9, 41-800 Zabrze, Poland, tel: +48 32 3733682, e-mail: karzab@sum.edu.pl

Received: 19.06.2018 Accepted: 3.09.2018 Available as AoP: 11.09.2018

Kardiologia Polska Copyright (c) Polish Cardiac Society 2018 


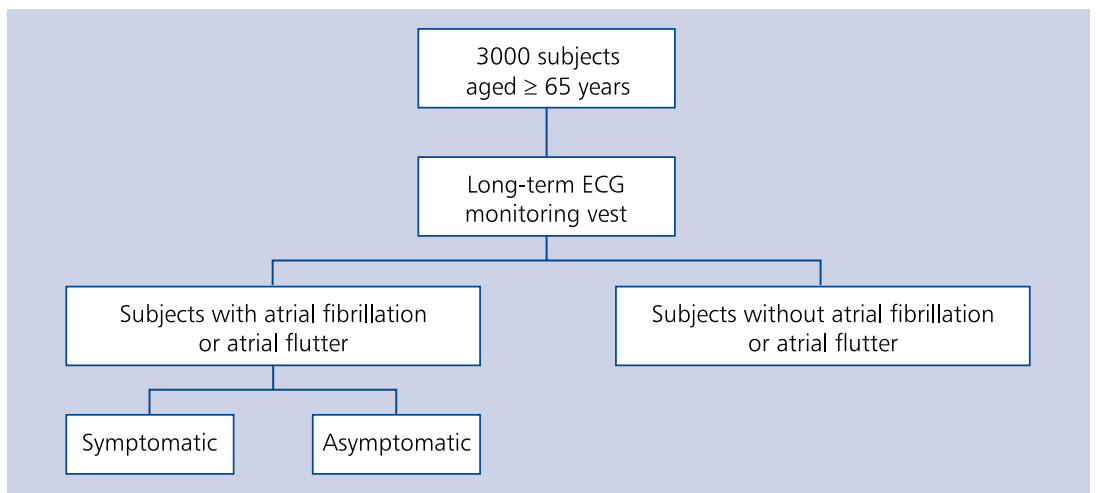

Figure 1. Study workflow; ECG — electrocardiogram

Table 1A. Schedule of assessments

\begin{tabular}{|c|c|c|c|c|}
\hline \multirow[t]{4}{*}{ Parameters } & Visit 1 & Visit 2 & Visit 3 & Visit 4 \\
\hline & Home visit & After & After & After \\
\hline & & 10 days \pm 4 days & 30 days \pm 4 days & 1 year \pm 7 days \\
\hline & & Home visit & Home visit & Phone call \\
\hline Informed consent & $\sqrt{ }$ & & & \\
\hline $\begin{array}{l}\text { Monitoring NOMED ECG } \\
\text { system installation }\end{array}$ & $\sqrt{ }$ & & & \\
\hline ECG telemonitoring (PMP) & Continuously & Continuously & Continuously & \\
\hline Monitoring system return & & & $\sqrt{ }$ & \\
\hline Medical and social questionnaire & $\sqrt{ }$ & & & $\sqrt{ }$ \\
\hline Present status monitoring questionnaire & & $\sqrt{ }$ & $\sqrt{ }$ & $\sqrt{ }$ \\
\hline Adverse event monitoring questionnaire & & $\sqrt{ }$ & $\sqrt{ }$ & $\sqrt{ }$ \\
\hline Geriatric Depression Scale assessment & & $\sqrt{ }$ & & \\
\hline Blood pressure measurements & $\sqrt{ }$ & $\sqrt{ }$ & $\sqrt{ }$ & \\
\hline Heart rate measurement & $\sqrt{ }$ & $\sqrt{ }$ & $\sqrt{ }$ & \\
\hline Anthropometric measurements & $\sqrt{ }$ & & $\sqrt{ }$ & \\
\hline Blood and urine samples & & $\sqrt{ }$ & & \\
\hline
\end{tabular}

ECG — electrocardiogram; PMP — patient monitoring platform (Comarch Healhcare)

Table 1B. The first visit questionnaire

\section{Data collected in the questionnaire}

Demographic data (e.g. education, marital status, employment)

Clinical symptoms and diseases

Medications taken

Medical history

Awareness of cardiovascular risk factors

Family medical history in first-degree relatives

Detailed information on atrial fibrillation/flutter episodes

(symptoms, duration, treatment, hospitalisations)

Habits (smoking, drinking)

Physical activity

Knowledge about health, disease, and prevention

\section{Study questionnaires}

The contents of the first visit questionnaire are outlined in Table 1B. The standardised Geriatric Depression Scale assessment, planned for the second visit, consists of 15 questions. Additionally, the second visit questionnaire comprises 10 questions focused on AF symptoms, fasting status, and any adverse events during the use of the ECG monitoring system. The third visit questionnaire includes 20 questions and is similar to the second visit one, with the addition of questions regarding outpatient visits, hospitalisations, and changes in medications. On the third visit, the Mini-Mental State Examination is completed as well. Finally, the fourth phone visit questionnaire involves 30 questions and gathers a similar scope of data as the third visit one but is expanded for survival status. 
The levels of the following parameters are planned to be measured through laboratory tests performed from blood samples: sodium, potassium, chloride, total cholesterol, high-density lipoprotein cholesterol, low-density lipoprotein cholesterol (direct and calculated measurement), triglycerides, creatinine, estimated glomerular filtration rate (calculated according to the Modification of Diet in Renal Disease and Chronic Kidney Disease Epidemiology Collaboration), glucose, thyrotropin hormone, total bilirubin, aspartate aminotransferase, alanine aminotransferase, gamma glutamyl transpeptidase, alkaline phosphatase, haemoglobin A1c, high-sensitivity C-reactive protein, and $\mathrm{N}$-terminal pro-B-type natriuretic peptide. The levels of creatinine, albumin, and albumin-to-creatinine ratio are planned to be tested from collected urine samples.

\section{Follow-up chart}

The follow-up chart consists of two parts, the first concerned with $\mathrm{AF}$ and the second with adverse events during the follow-up period. The first part is to be filled out with data on symptomatology (European Heart Rhythm Association scale), approach to treatment (no treatment or rhythm/rate control), and methods used (drugs/ablation). The second part is designed to collect data on every potential adverse event, including unplanned hospitalisation, unscheduled outpatient visit, emergency service callout, or death, as well as its specifics (date, type, and a detailed description).

\section{Endpoints}

The primary endpoint is the prevalence of $\mathrm{AF}$, especially the silent type, in Polish individuals aged $\geq 65$ years. The secondary endpoint is to determine the independent risk factors for the occurrence of $\mathrm{AF}$, especially the silent type. Additional endpoints include the prevalence of heart failure, stroke, hypertension, and vascular diseases.

\section{Monitoring system}

The non-invasive, long-term ECG monitoring system used in the study was developed by Comarch Healthcare (Krakow, Poland) based on the GSM technology. This system comprises a mobile long-term ECG vest with dry silver ECG electrodes, exchangeable recorders fixed to the vest, a docking station acting as a charger, a GSM transmitter, and a patient monitoring platform for data analysis and storage. All study participants will be equipped with two recorders to maintain continuous ECG acquisition during charging and enable the transmission of data to the monitoring platform every $24 \mathrm{~h}$. Participants will be trained on how to use the ECG monitoring device at the first visit by the study nurse. For further details refer to Supplement on-line (see journal website).

\section{Statistical analysis}

As the study will analyse equivalent age strata, the sample will not reflect the structure of the elderly population (age $\geq 65$ years). After the end of the study, it is planned to calculate weighted adjustments for epidemiological analyses. Statistical analyses of the data will be performed using SAS software for Windows (version 9.4, Cary, NC, USA) and $\mathrm{R}$ software for statistical computing (The R Foundation). Basic statistical analyses involving estimation of the indicators defined in the study objectives will be conducted using the SURVEYFREQ and SURVEYMEANS procedures of the SAS package, considering the complex sample and design.

\section{DISCUSSION}

Current guidelines recommend that AF screening be provided for all symptomatic individuals and for those who are asymptomatic but have a high risk for thromboembolism. AF screening may be performed using simple methods, e.g. pulse palpation or, in those with cardiac implanted electronic devices, using high atrial rate analysis during interrogation. Nevertheless, the AF diagnosis should always be confirmed by a standard ECG recording $[7,8]$.

A short-lasting, asymptomatic episode of AF may result in a 2.8-fold higher risk of ischaemic stroke and a 2.5-fold higher risk of fatality [9]. Among elderly individuals, episodes of arrhythmia are often mildly symptomatic or completely asymptomatic, which may significantly hinder the possibility of diagnosing the arrhythmia [10]. Fernandez et al. [11] showed that 24-h monitoring of individuals with acute-phase ischaemic stroke enables AF detection in as many as $10 \%$ of hospitalised patients. Grond et al. [3] determined that monitoring of individuals with a recent (occurring within the last $72 \mathrm{~h}$ ) ischaemic stroke is in direct correlation with a higher AF detection rate and thus results in earlier introduction of optimal treatment and prevention. Early results of the PROFIL-AF study indicate that the simple action of taking the pulse and interviewing individuals regarding symptoms suggesting arrhythmia enables AF detection, including an additional 3.4\% of individuals aged $>65$ years who had never before been diagnosed with AF. Studies reporting the incidence of silent AF have been summarised in Supplemental Table 1 (see journal website).

Such high detection rates were based on single tests performed in individuals, therefore it may be assumed that long-term monitoring would facilitate AF detection in larger groups. Episodes of clinically silent AF are strictly correlated with a higher frequency of a so-called silent stroke [12], as detected based on nuclear magnetic resonance imaging, with a prevalence of $8 \%$ to $28 \%$. The main causes of silent stroke are AF (28\%) and hypertension [13]. The silent stroke doubles the risk of dementia, especially among elderly individuals [14].

Thus, asymptomatic AF is an important and underestimated clinical issue. A correct and early diagnosis of arrhythmia would enable the introduction of the correct treatment and thereby would help to prevent thromboembolic complications by the use of oral anticoagulants [15]. Improvements in 
the field of monitoring and detecting arrhythmia through the implementation of simple, easy-to-use, non-invasive devices capable of registering electric activity of the heart, performing preliminary rhythm analysis, and enabling long-term monitoring and remote data transmission may facilitate the diagnostic process.

In conclusion, the NOMED-AF study will determine the true proportion of individuals with asymptomatic AF and help to ascertain the independent factors predisposing to this arrhythmia. The study will identify the population of older individuals requiring AF screening.

Funding: The research has received funding from the National Centre for Research and Development under grant agreement (STRATEGMED2/269343/18/NCBR/2016).

\section{Conflict of interest: none declared}

\section{References}

1. Israel CW, Grönefeld G, Ehrlich JR, et al. Long-term risk of recurrent atrial fibrillation as documented by an implantable monitoring device: implications for optimal patient care. J Am Coll Cardiol. 2004; 43(1): 47-52, indexed in Pubmed: 14715182.

2. Lau JK, Lowres N, Neubeck L, et al. iPhone ECG application for community screening to detect silent atrial fibrillation: a novel technology to prevent stroke. Int J Cardiol. 2013; 165(1): 193-194, doi: 10.1016/j.ijcard.2013.01.220, indexed in Pubmed: 23465249.

3. Grond M, Jauss M, Hamann G, et al. Improved detection of silent atrial fibrillation using 72-hour Holter ECG in patients with ischemic stroke: a prospective multicenter cohort study. Stroke. 2013; 44(12): 3357-3364, doi: 10.1161/STROKEAHA.113.001884, indexed in Pubmed: 24130137.

4. Healey JS, Alings M, Ha A, et al. ASSERT-II Investigators. Subclinical atrial fibrillation in older patients. Circulation. 2017; 136(14): 1276-1283, doi: 10.1161/CIRCULATIONAHA.117.028845, indexed in Pubmed: 28778946.

5. Zhao J, Hansen BJ, Wang Y, et al. Three-dimensional integrated functional, structural, and computational mapping to define the structural "fingerprints" of heart-specific atrial fibrillation drivers in human heart ex vivo. J Am Heart Assoc. 2017; 6(8), doi: 10.1161/JAHA.117.005922, indexed in Pubmed: 28862969.

6. Quinn FR, Gladstone D. Screening for undiagnosed atrial fibrillation in the community. Curr Opin Cardiol. 2014; 29(1):
28-35, doi: 10.1097/HCO.0000000000000018, indexed in Pubmed: 24281348 .

7. Mairesse GH, Moran P, Van Gelder IC, et al. Screening for atrial fibrillation: a European Heart Rhythm Association (EHRA) consensus document endorsed by the Heart Rhythm Society (HRS), Asia Pacific Heart Rhythm Society (APHRS), and Sociedad Latinoamericana de Estimulación Cardíaca y Electrofisiología (SOLAECE). Europace. 2017; 19(10): 1589-1623, doi: 10.1093/europace/eux177, indexed in Pubmed: 29048522.

8. Freedman B, Camm J, Calkins H, et al. Screening for atrial fibrillation. A Report of the AF-SCREEN International Collaboration. Circulation. 2017; 135(19): 1851-1867, doi: 10.1161/circulationaha.116.026693.

9. Dobreanu D, Svendsen JH, Lewalter T, et al. Current practice for diagnosis and management of silent atrial fibrillation: results of the European Heart Rhythm Association survey. Europace. 2013; 15(8): 1223-1225, doi: 10.1093/europace/eut227, indexed in Pubmed: 23878150.

10. Friberg L, Engdahl J, Frykman V, et al. Population screening of 75- and 76-year-old men and women for silent atrial fibrillation (STROKESTOP). Europace. 2013; 15(1): 135-140, doi: 10.1093/europace/eus217, indexed in Pubmed: 22791299.

11. Fernandez V, Béjot Y, Zeller M, et al. Silent atrial fibrillation after ischemic stroke or transient ischemic attack: interest of continuous ECG monitoring. Eur Neurol. 2014; 71(5-6): 313-318, doi: 10.1159/000357561, indexed in Pubmed: 24685765.

12. Cha MJ, Park HE, Lee MH, et al. Prevalence of and risk factors for silent ischemic stroke in patients with atrial fibrillation as determined by brain magnetic resonance imaging. Am J Cardiol. 2014; 113(4): 655-661, doi: 10.1016/j.amjcard.2013.11.011, indexed in Pubmed: 24360776.

13. Vermeer SE, Prins ND, den Heijer T, et al. Silent brain infarcts and the risk of dementia and cognitive decline. N Engl J Med. 2003; 348(13): 1215-1222, doi: 10.1056/NEJMoa022066, indexed in Pubmed: 12660385.

14. Park JW, Camm AJ, Lip GYH, et al. 2012 focused update of the ESC Guidelines for the management of atrial fibrillation: an update of the 2010 ESC Guidelines for the management of atrial fibrillation. Developed with the special contribution of the European Heart Rhythm Association. Eur Heart J. 2012; 33(21): 2719-2747, doi: 10.1093/eurhearti/ehs253, indexed in Pubmed: 22922413.

15. Lip GYH, Laroche C, Dan GA, et al. A prospective survey in European Society of Cardiology member countries of atrial fibrillation management: baseline results of EURObservational Research Programme Atrial Fibrillation (EORP-AF) Pilot General Registry. Europace. 2014; 16(3): 308-319, doi: 10.1093/europace/eut373, indexed in Pubmed: 24351881.

Cite this article as: Kalarus Z, Balsam P, Bandosz P, et al. NOninvasive Monitoring for Early Detection of Atrial Fibrillation: rationale and design of the NOMED-AF study. Kardiol Pol. 2018; 76(10): 1482-1485, doi: 10.5603/KP.a2018.0193. 